\title{
Awake proning in COVID-19 — does CPAP make a difference?
}

\section{To the Editor}

Awake proning is being used as an adjunct to the current therapies for patients with cooronavirus disease 2019 (COVID-19) related hypoxemic respiratory failure. Although there is no universally accepted protocol, there is emerging data on the use of awake prone positioning [1,2]. Although most studies have described patients on CPAP, high flow nasal cannula as well as standard oxygen therapies in different proportions, the response of prone positioning may likely be different among these as the application of positive end-expiratory pressure may lead to better recruitment, which is one of the major mechanism of the effect of prone positioning. Coppo et al., in a recent issue of The Lancet Respiratory Medicine, have reported results of a prospective study to assess the effects of awake proning in patients with COVID-19 [3]. This is the largest study till date regarding the utility of awake proning, in which the authors compared the characteristics of patients who responded to awake proning with those who did not and found the time between hospital admission and prone positioning to be the only variable significantly different between the groups. The one thing missing in the analysis in this study was whether the number of patients receiving continuous positive airway pressure (CPAP) and conventional oxygen therapy was similar between the groups. As CPAP might correct hypoxemia more than standard oxygen delivery, it will be interesting to know whether patients receiving CPAP therapy were more in the responders. On the literature review, we found only two other studies which have reported the use of awake proning in COVID-19 patients on continuous positive pressure ventilation, and both included patients on non-invasive positive pressure ventilation. Sartini et al. reported the use of prone positioning outside ICU in 15 patients on NIV and documented significant improvement in oxygenation, although absolute changes were not reported [4]. On the other hand, Golestanieraghi et al. reported results from 10 patients and had an increase in mean $\mathrm{PO} 2$ from 46.34 to $62.54 \mathrm{mmHg}$ after proning [5]. Other studies have described the use of prone positioning in patients on conventional oxygen therapy or HFNC [6]. The effect of CPAP on oxygenation parameters during awake proning in patients with COVID-19-related ARDS is yet to be determined, and further studies are required to assess the same as this strategy may be a game changer for the ongoing COVID-19 pandemic.

\section{Conflict of interest}

None declared.

\section{References:}

1. Sryma PB, Mittal S, Madan K, et al. Reinventing the Wheel in ARDS: Awake Proning in COVID-19. Arch Bronconeumol. 2020; 56(11): 747-749, doi: 10.1016/j.arbres.2020.06.002, indexed in Pubmed: 32736877.

2. Bamford P, Bentley A, Dean J, et al. ICS Guidance for Prone Positioning of the Conscious COVID Patient. 2020.

3. Coppo A, Bellani G, Winterton D, et al. Feasibility and physiological effects of prone positioning in non-intubated patients with acute respiratory failure due to COVID-19 (PRONCOVID): a prospective cohort study. Lancet Respir Med. 2020; 8(8): 765-774, doi: 10.1016/S2213-2600(20)30268-X, indexed in Pubmed: 32569585.

4. Sartini C, Tresoldi M, Scarpellini P, et al. Respiratory parameters in patients with COVID-19 after using noninvasive ventilation in the prone position outside the intensive care unit. JAMA. 2020; 323(22): 2338-2340, doi: 10.1001/ jama.2020.7861, indexed in Pubmed: $\underline{32412606 .}$.

5. Golestani-Eraghi M, Mahmoodpoor A. Early application of prone position for management of Covid-19 patients. J Clin Anesth. 2020; 66: 109917, doi: 10.1016/j.jclinane.2020.109917, indexed in Pubmed: 32473503.

6. Elharrar X, Trigui Y, Dols AM, et al. Use of prone positioning in nonintubated patients with COVID-19 and hypoxemic acute respiratory failure. JAMA. 2020; 323(22): 2336-2338, doi: 10.1001/jama.2020.8255, indexed in Pubmed: 32412581.

Address for correspondence: Saurabh Mittal, All India Institute of Medical Sciences, Ansari Nagar, Delhi, India; e-mail: saurabh_kgmu@yahoo.co.in

DOI: 10.5603/ARM.a2020.0179

Received: 07.08.2020

Copyright (C) 2021 PTChP

ISSN 2451-4934 